\title{
Análisis energético de la biomasa en Michoacán: Con caso de estudio para generación eléctrica de $4 \mathrm{MWh}$
}

\author{
Biomass energy analysis in Michoacán: With study case for 4 MWh electric \\ generation
}

\author{
ZAPIEN-RODRÍGUEZ, José Manuel†*, ESCOTO-SOTELO, Edgardo Abdiel y NÚÑEZ-PÉREZ, \\ Francisco Augusto
}

Universidad Politécnica de Lázaro Cárdenas, Ingeniería Mecatrónica Av. Galeanas Sin Número, Colonia Las 600 Casas, Cd. Lázaro Cárdenas, Michoacán, C.P. 60950

ID $1^{\mathrm{er}}$ Autor: José Manuel, Zapien-Rodríguez / ORC ID: 0000-0001-7198-2118, CVU CONACYT ID: 228342

ID $1^{\text {er }}$ Coautor: Edgardo Abdiel, Escoto-Sotelo / ORC ID: 0000-0002-9802-9770, CVU CONACYT ID: 390313

ID $2^{\text {do }}$ Coautor: Francisco Augusto, Núñez-Pérez

DOI: $10.35429 /$ JEE.2019.9.3.29.34

Recibido Marzo 20, 2019; Aceptado Junio 30, 2019

\begin{abstract}
Resumen
La reforma energética promueve el uso de energías renovables para hacer frente a la reducción del consumo de energías fósiles. Michoacán es un estado donde prolifera la agricultura, contando con una diversidad de climas que favorece al cultivo de una variedad significativa de frutos, además que su área de cosecha es abundante. En muchas ocasiones los residuos generados de la cosecha, representa un problema para los agricultores, debido a que el volumen es demasiado y no saben que uso darle, desafortunadamente optan por la quema ilegal de dichos residuos. Sin embargo, la biomasa puede usarse como combustible con rendimientos térmicos elevados, con un costo inferior al de otros combustibles, lo que representa una alternativa para numerosas industrias que requieren combustión en sus procesos y servicios. Michoacán destaca continuamente en los primeros lugares en la producción de diversos alimentos a nivel nacional, pero sus residuos se emplean para el consumo en ganado, por lo que este trabajo de investigación no solo toma en cuenta la cosecha neta, si no que realiza un estimado de residuos efectivos que pueden considerarse biomasa disponible para su uso en procesos térmicos, indicando de acuerdo a su poder calorífico el potencial energético hasta el momento desaprovechado.
\end{abstract}

Biomasa, Análisis Energético, Generación Eléctrica

\begin{abstract}
The energy reform promotes the use of renewable energies to face the reduction of fossil energy consumption. Michoacán is a state where agriculture proliferates, counting on a diversity of climates that favors the cultivation of a significant variety of fruits, in addition that its harvest area is abundant. On many occasions the waste generated from the harvest represents a problem for the farmers, because the volume is too much and they do not know what to do, unfortunately they opt by the illegal burning. However, biomass can be used as fuel with high thermal outputs, with a lower cost than other fuels, which represents an alternative for many industries that require combustion in their processes. Michoacán stands out continuously at the first places in the production of diverse fruts at national level, but its residues are used only for cattle consumption, so this research work not only takes into account the net harvest, it also makes an estimate of effective waste that can be considered biomass available for use in thermal processes, indicating according to its calorific value the energy potential wasted until the moment.
\end{abstract}

Biomass, Energy Analysis, Electric Generation

Citación: ZAPIEN-RODRÍGUEZ, José Manuel, ESCOTO-SOTELO, Edgardo Abdiel y NÚÑEZ-PÉREZ, Francisco Augusto. Análisis energético de la biomasa en Michoacán: Con caso de estudio para generación eléctrica de 4MWh. Revista de Ingeniería Eléctrica. 2019. 3-9: 29-34

\footnotetext{
$\dagger$ Investigador contribuyendo como primer Autor.

*Correspondencia al Autor correo electrónico:(zapien_jomazaro@uplc.edu.mx)
} 


\section{Introducción}

México, así como muchos países cuenta con problemas ambientales debido a la dependencia energética de hidrocarburos, debido al elevado consumo de combustibles fósiles, siendo necesario el desarrollo de diferentes modelos energéticos renovables, sostenibles, eficientes, económicamente viables, prácticos y seguros, que den respuesta a las necesidades de abastecimiento de energía en el contexto de un desarrollo social sostenible.

La biomasa juega un papel importante en el nuevo marco energético federal, debido a que los residuos agrícolas que se producen en todo México son relativamente grande, sin embargo la eficiencia energética que presentan pueden variar de acuerdo a las características de cada producto. Los combustibles agrícolas se clasifican según la FAO (Organización de las Naciones Unidas para la Agricultura y la Alimentación) en cultivos de combustible, subproductos agrícolas (paja, tallos), los subproductos animales (estiércol y otros excrementos) y subproductos agroindustriales.

La biomasa analizada a diferencia de una cosecha agrícola deberá delimitarse a residuos, no a la producción total, además se debe considerar que estos residuos en muchas ocasiones son empleados como alimento de ganado, por lo que se considera un residuo agrícola de cosecha (RAC) de $2^{\text {da }}$ generación para el análisis de eficiencia energética de la biomasa, además que a lo largo de todo un año no se cuentan con una misma cosecha ya que es de acuerdo a la temporada de producción.

En la región occidente de México, la condiciones climatológicas son propicias para la cosecha de una gran variedad de flora, puntualmente Michoacán se considera líder mundial en la producción de Aguacate, sin embargo este no es el único alimento con alto impacto de cosecha en este estado. De acuerdo al información obtenida por el Desarrollo Rural y Agroalimentario (SEDRUA), Michoacán cuenta con 20 productos que ocupan el primer lugar de producción en el país, entre los cuales se encuentran el aguacate, guayaba, lenteja, fresa, zarzamora, fresa, camote, carambolo, forrajes, mano de león (flor); cempazúchil y forrajes, así como el limón, toronja, pera, lima, garbanzo, col, chayote, alcachofa, chile perón con buena producción agrícola.

\section{La energía en la biomasa}

Todo material que contenga carbón en su estructura molecular es viable a usarse como combustible, proporcionando una cantidad de energía acorde a su poder calorífico. La energía obtenida a través del uso de la biomasa, es básicamente utilizando la materia orgánica como fuente energética.

Esta materia orgánica, es heterogénea y puede usarse de forma directa o peletizada. Su utilizan es mediante calderas donde la biomasa se quema poco a poco, quedando como residuo solo ceniza, la cual se puede utilizar como abono.

El empleo de la biomasa como fuente alternativa de energía limpia ha tenido auge debido a una serie de factores, tales como:

- Incremento de precios de los productos derivados del petróleo.

- Crecimiento de la producción agrícola.

- $\quad$ El cambio climático.

- Aumento de la preparación técnica y el conocimiento científico en la investigación de renovables.

- Escenario económico propicio para la explotación de plantas de biomasa.

Trabas burocráticas a otro tipo de promoción de energías renovables.

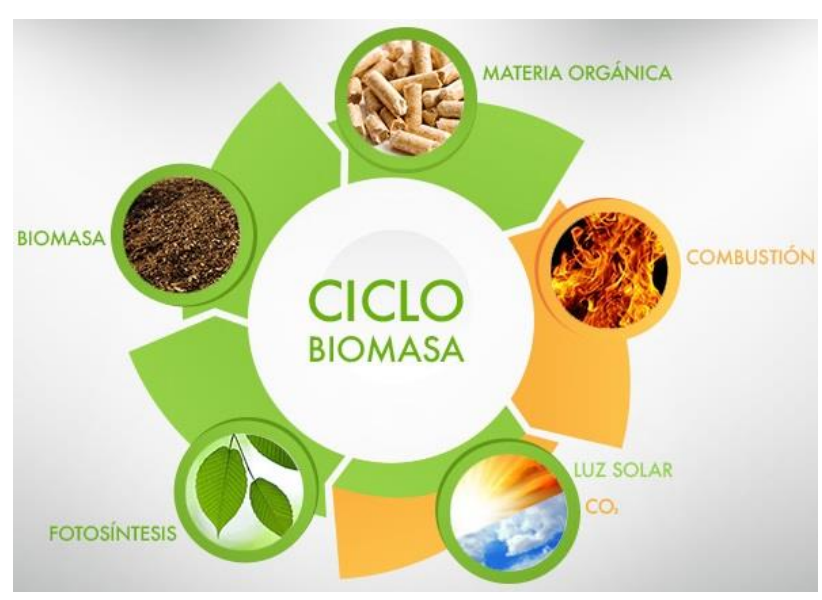

Figura 1 Ciclo energético de la Biomasa

Fuente: https://www.cointra.es

De forma casi directa se asocia a la biomasa con el bagazo de la caña, trigo, maíz y sorgo, o en su defecto a la combinación de estos, debido a que son los productos que cuentan con una mayor cantidad de residuos una vez extraído su fruto. 
Del punto de vista energético la Biomasa considera cuatro características importantes para determinar su poder calorífico: Carbón Fijo, Materia Volátil, Ceniza y Humedad.

La composición química de la biomasa es compleja; los componentes primordiales incluyen seis elementos principales $\mathrm{C}, \mathrm{H}, \mathrm{O}, \mathrm{N}$, $\mathrm{S}, \mathrm{Cl}$ y al menos otros ocho elementos $\mathrm{Si}, \mathrm{Al}, \mathrm{Ca}$, $\mathrm{K}, \mathrm{Mg}, \mathrm{Na}, \mathrm{P}$ y Fe.

El principal componente de la biomasa es el C, con un proporción del $30 \%$ al $60 \%$ en peso de materia seca. El segundo elemento en importancia, con un $30 \%$ a $40 \%$ es el Oxígeno. El tercer componente principal es el $\mathrm{H}$, que comprende normalmente de $5 \%$ a $6 \%$ en peso de la materia seca.

Mientras que el $\mathrm{N}$, el $\mathrm{S}$ y el $\mathrm{Cl}$ suelen ser menos del $1 \%$ del peso de la biomasa. Estos valores se determinaron con un porcentaje de humedad por debajo del $11 \%$.

La combustión es la oxidación rápida de un combustible $(\mathrm{Cx} \mathrm{Hy} \mathrm{Oz}$ ) resultando en un gas no combustible, esto se realizar a través de las siguientes etapas:

$\mathrm{C}+\mathrm{O}_{2} 2=\mathrm{CO}_{2}+$ calor

Secado: Vaporización de agua

Combustible + calor $=$ Combustible + vapor de agua

Pirolisis/gasificación: Degradación térmica en la ausencia o presencia de oxígeno suministrado externamente.

Combustible sólido + Calor $=$ Gases

Combustión: Oxidación del carbón - oxidación final de los gases de carbón y combustión

Combustible $+\mathrm{O}_{2} \rightarrow \mathrm{CO}_{2}+\mathrm{H}_{2} \mathrm{O}+$ Energia

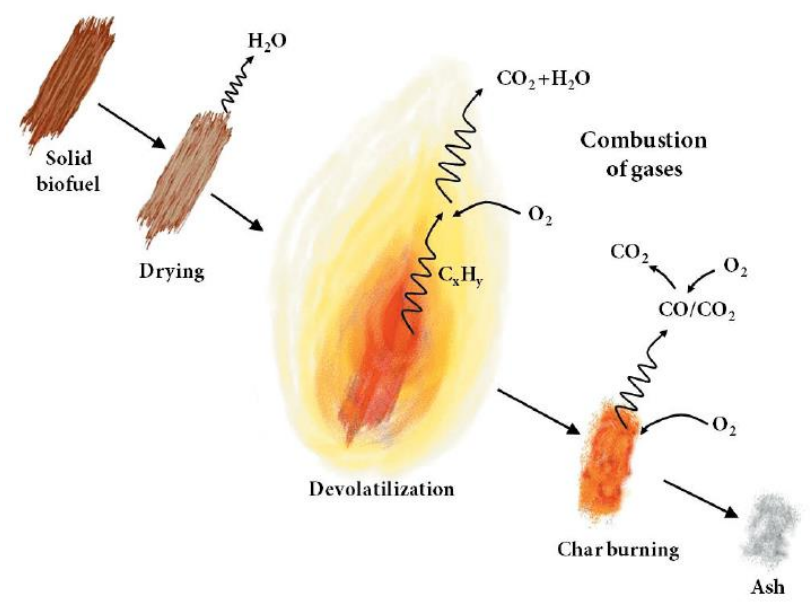

Figura 2 Ciclo de Combustión

Fuente: https://www.researchgate.net

\section{Comparativa Calorífica y económica entre Biomasa y Carbón}

El carbón es de los hidrocarburos fósiles que se emplean con mayor frecuencia tanto de manera domestica como industrial, donde su uso esencial es aprovechar su poder calorífico en la combustión.

Los componentes químicos de la Biomasa con una humedad por debajo del $11 \%$ que determina el PCI es el porcentaje de Carbón Fijo, Material Volátil y la Ceniza. Comparando las propiedades de la biomasa con el carbón mineral se tiene que:

\begin{tabular}{|l|r|r|r|}
\hline \multicolumn{1}{|c}{$\%$} & Ceniza & \multicolumn{1}{r|}{ Volátil } & Carbón Fijo \\
\hline Sorgo & 17 & 62 & 21 \\
\hline Maíz & 2,1 & 78,9 & 19,05 \\
\hline Trigo & 2,8 & 80 & 17,2 \\
\hline Cebada & 6,1 & 77,9 & 16 \\
\hline Paja & 6,2 & 82 & 11,9 \\
\hline Carbón M. & 4,5 & 18 & 77 \\
\hline
\end{tabular}

Tabla 1 Composición de Biomasas y Carbón Mineral

El Poder Calorífico Inferior (PCI) del carbón mineral es de alrededor de $6300 \mathrm{kCal} / \mathrm{kg}$, por lo que en una Tonelada se tendría una energía 26,520 MJ/Ton.

Una vez que la biomasa se somete previamente a un proceso de secado y triturado para mantener una humedad por debajo del $11 \%$, cuenta con un PCI de $3700 \mathrm{kCal} / \mathrm{kg}$ a 4300 $\mathrm{kCal} / \mathrm{kg}$, que corresponde en energía a 15.5 $\mathrm{MJ} / \mathrm{kg}$ a $18 \mathrm{MJ} / \mathrm{kg}$. Por lo que para suministrar la misma energía del carbón mineral se requiere de 1.5 a 1.7 veces más de Biomasa por cada cantidad de Carbón. 
El precio del carbón mineral colombiano por tonelada de acuerdo al World Bank, es de 74 USD, mientras que si se considera a la Biomasa como combustible, los costos de adquisición dependen de múltiples factores tales como la fuente, la oferta y demanda, contenido de humedad, transporte y procesamiento, sin embargo debido a que deshacerse de estos residuos puede representar un costo para los agricultores.

El valor de los residuos de $2 \mathrm{da}$ generación esta del orden de 2 a 5 USD/TON, sin embargo, su costo puede incrementar en caso que se tenga demanda de la biomasa como materia prima para la industria de la celulosa alcanzando hasta los 20 y 40 USD/TON según el tipo de residuo forestal. Finalmente el costo del flete se determina usando una distancia media de entre 50 y $70 \mathrm{~km}$ con un costo asociado de 0,12 y $0,20 \mathrm{USD} / \mathrm{TON} / \mathrm{km}$.

\section{Caso de estudio: Relación Energía - Potencia}

La energía es la capacidad que se necesita para realizar trabajo. Teóricamente la palabra trabajo es el producto de multiplicar fuerza por distancia. Por ejemplo, si se eleva un cuerpo que pesa un kilogramo a una altura de un metro, el trabajo realizado es numéricamente igual a un kilogramo-metro. Por su parte, el trabajo realizado en un determinado tiempo se define como potencia. Así $1 \mathrm{~J}$ de energía es aplicada durante $1 \mathrm{~s}$, es decir, $100 \mathrm{~g}$ levantados $1 \mathrm{~m}$ en $1 \mathrm{~s}$, entonces la potencia requerida es exactamente 1 watt (W). La caloría está definida en términos de energía calórica como el calor requerido para incrementar $1{ }^{\circ} \mathrm{C}$ la temperatura de $1 \mathrm{~g}$ de agua, que es equivalente a $4.18 \mathrm{~J}$. De manera analítica la relación que existe entre calorías y Wh, proviene de las siguientes equivalencias:

$$
1 \mathrm{cal}=4.19 \mathrm{~J} \quad \mathrm{y} \quad 1 \mathrm{kWh}=3.6 \mathrm{MJ}
$$

Por lo tanto: $1 \mathrm{kWh}=859.85 \mathrm{kCal}$

Considerando que el poder calorífico del carbón es de $6300 \mathrm{kCal} / \mathrm{kg}$ que corresponden a $26.397 \mathrm{MJ} / \mathrm{kg}$, la conversión ideal a potencia en Wh sería de $7.332 \mathrm{kWh} / \mathrm{kg}$, cuando el rango de consumo residencial bajo de CFE es menor a 450 $\mathrm{kWh}$ bimestrales, lo que equivale a que una familia para permanecer en la cuota de bajo consumo podrá disponer de hasta 61.37 Kilogramos de Carbón bimestrales.
En caso que la potencia eléctrica que requiere la familia sea suministrada exclusivamente por biomasa cuyo PCI sería de $4300 \mathrm{kCal} / \mathrm{kg}$ equivalente a $18 \mathrm{MJ} / \mathrm{kg}$ (considere el tratamiento previo al cual deberá ser sometido para alcanzar dichos valores de PCI), la biomasa tendrá una potencia de $5 \mathrm{kWh} / \mathrm{kg}$, de esta manera para alcanzar el límite de $450 \mathrm{kWh}$ bimestrales permitidos para el Rango Bajo se requiere la energía de 90 Kilogramos de Biomasa.

De esta manera la ecuación general obtenida para relacionar la Potencia Eléctrica Consumida (PEC) y Cantidad de Combustible (CC) necesaria es:

$C C=\frac{P E C(k W h) * 859.85(k C a l / k W h)}{P C I(k C a l / k g)}$

Dónde:

$\mathrm{CC}=$ Cantidad de Combustible $(\mathrm{kg})$

$\mathrm{PEC}=$ Potencia Eléctrica Consumida (kWh)

PCI = Poder Calorífico Inferior del Combustible $(\mathrm{kCal} / \mathrm{kg})$

\section{Discusiones}

El caso de estudio analizado anteriormente revela una cantidad de biomasa bimestral alcanzable, recientemente la UdeG inauguró su primer huerto solar con una inversión de 20 millones de pesos con una generación eléctrica de 4.01 MWh, equivalentes a reducir 2 Toneladas de emisiones de $\mathrm{CO} 2$ anuales. Asi mismo para satisfacer esta generación eléctrica usando Biomasa con un poder calorífico de 4300 $\mathrm{kCal} / \mathrm{kg}$ se requeriría de:

$C C=\frac{P E C(k W h) * 859.85(k C a l / k W h)}{P C I(k C a l / k g)} \approx 802^{k g} / \mathrm{hr}$

Por lo que cronológicamente se deberá mantener un abastecimiento de biomasa de 802 $\mathrm{kg} / \mathrm{hr}$ por lo que en un día se deberá tener 19.248 Ton/día, esto bajo condiciones de Eficiencia al $100 \%$, y tratamiento previo a la biomasa.

\begin{tabular}{|l|r|}
\hline Tiempo & Peso (Ton) \\
\hline 1 hora & 0,802 \\
\hline 1 día & 19,248 \\
\hline 1 año & 7025,52 \\
\hline
\end{tabular}

Tabla 2 Abastecimiento de Biomasa con Eficiencia Térmica al $100 \%$ y PCI de $4300 \mathrm{kCal} / \mathrm{kg}$ 
En condiciones reales de operación, la eficiencia térmica de las calderas es del $80 \%$, además que la Biomasa de 2da Generación contemplada cuenta con un poder calorífico inferior del orden $3700 \mathrm{kCal} / \mathrm{kg}$, el abastecimiento de Biomasa se incrementa hasta un $45 \%$ la cantidad de combustible requerido:

\begin{tabular}{|l|r|}
\hline Tiempo & Peso (Ton) \\
\hline 1 hora & 1,164 \\
\hline 1 día & 27,936 \\
\hline 1 año & 10196,64 \\
\hline
\end{tabular}

Tabla 3 Abastecimiento de Biomasa con Eficiencia Térmica al $80 \%$ y PCI de $3700 \mathrm{kCal} / \mathrm{kg}$

Finalmente para tener mantener el comparativo Biomasa - Carbón, la cantidad de Carbón requerido en condiciones reales (Eficiencia Térmica al 80\%), se verá reflejado en:

\begin{tabular}{|l|r|}
\hline Tiempo & Peso (Ton) \\
\hline 1 hora & 0,684 \\
\hline 1 día & 16,416 \\
\hline 1 año & 5991,84 \\
\hline
\end{tabular}

Tabla 4 Abastecimiento de Carbón Mineral con Eficiencia Térmica al $80 \%$ y PCI de $6300 \mathrm{kCal} / \mathrm{kg}$

Por lo tanto para dejar de lado un aproximado de 16.5 Toneladas de Carbón Mineral por día, se requiere un cantidad de aproximadamente 28 Toneladas de Biomasa de 2da Generación diaria.

\section{Conclusiones}

El uso de biomasa como biocombustible ha sido ampliamente utilizado, ya que cuenta con diversas ventajas económicas y ambiéntalas comparada con los hidrocarburos fósiles. Su utilización se enfoca a pequeña escala, debido a que al aumentar la demanda de la biomasa se complica su abastecimiento continuo.

El principal problema es el abastecimiento de la biomasa y su tratamiento previo para reducir su porcentaje de humedad y así aumentar su densidad y poder calorífico, inclusive se puede emplear la Pirolisis para fijar el Carbón de la Biomasa y eliminar una gran parte de Materia Volátil, logrando que su PCI aumente al mismo nivel del Carbón Mineral.
En la mayoría de los casos el uso el uso de la Biomasa como combustible se hace en instalaciones hibridas, para complementar y asegurar el abastecimiento de la materia prima.

De igual forma actualmente se están realizando estudios en la misma Universidad Politécnica de Lázaro Cárdenas (UPLC) con Biomasa modificada para incrementar la cantidad de producto en espacios reducidos a los naturalmente requeridos, teniendo resultados muy favorables.

\section{Referencias}

Agüero, A. C., Pisa, J. R., Agüero, C. J., \& Bugeau, A. T. (2010). Poder calorífico del bagazo de caña de azúcar. Junio

Bustamante, C. A. G., \& Cerutti, O. M. (2017). Estado del arte de la bioenergía en México.

Carpenter, D., Westover, T. L., Czernik, S., \& Jablonski, W. (2014). Biomass feedstocks for renewable fuel production: a review of the impacts of feedstock and pretreatment on the yield and product distribution of fast pyrolysis bio-oils and vapors. Green Chemistry, 16(2), 384-406.

Golato, M. A., Franck Colombres, F. J., Aso, G., Correa, C. A., \& Paz, D. (2008). Metodología de cálculo de la eficiencia térmica de generadores de vapor. Revista industrial y agrícola de Tucumán, 85(2), 17-31.

Hernández Sardiñas, A., González Morales, V. M., \& Freide Orozco, M. L. (2017). Aprovechamiento de las posibles fuentes de biomasa para entregar más electricidad en la fábrica de azúcar antonio sánchez. Centro Azúcar, 44(4), 88-97.

Jenkins, B., Baxter, L. L., Miles Jr, T. R., \& Miles, T. R. (1998). Combustion properties of biomass. Fuel processing technology, 54(1-3), $17-46$.

Masera, O., Coralli, F., García, C., Riegelhaupt, E., Arias, T., Vega, J., \& Cecotti, L. (2011). La bioenergía en México. Situación actual y perspectivas. Red Mexicana de Bioenergía, AC, México, 7-8. 
McKendry, P. (2002). Energy production from biomass (part 1): overview of biomass. Bioresource technology, 83(1), 37-46.

Ordóñez Díaz, J. A. B., Rivera Vázquez, R., Tapia Medina, M. E., \& Ahedo Hernández, L. R. (2015). Contenido y captura potencial de carbono en la biomasa forestal de San Pedro Jacuaro, Michoacán. Revista mexicana de ciencias forestales, 6(32), 7-16.

Raveendran, K., Ganesh, A., \& Khilar, K. C. (1996). Pyrolysis characteristics of biomass and biomass components. Fuel, 75(8), 987-998.

Rueda, G. D. H. Z., Mistretta, M. G., Peralta, F. L., Gutiérrez, C. E., Feijóo, E. A., Golato, M. A., ... \& Cárdenas, G. J. (2016). CARACTERIZACIÓN ENERGÉTICA DEL RESIDUO AGRÍCOLA DE COSECHA DE CAÑA DE AZÚCAR (RAC) DE TUCUMÁN, ARGENTINA. Energías Renovables y Medio Ambiente (ERMA), 36.

Serrano, J., Mejía, W., Ortiz, J., Sánchez, A., \& Zalamea, S. (2017). Determinación del potencial de generación eléctrica a partir de biomasa en el Ecuador.

Servicio de Información Agroalimentaria y Pesquera (SIAP). (2009). Consultado 09-112010 en http://www.siap.sagarpa.gob.mx/

SOLAR, E. T. (2016). CENTRO INTERDISCIPLINARIO DE INVESTIGACIÓN PARA EL DESARROLLO INTEGRAL REGIONAL CIIDIR MICHOACÁN (Doctoral dissertation, INSTITUTO POLITÉCNICO NACIONAL).

Vassilev, S. V., Vassileva, C. G., \& Vassilev, V. S. (2015). Advantages and disadvantages of composition and properties of biomass in comparison with coal: An overview. Fuel, 158, 330-350. 